\title{
Desenvolvimento neuropsicomotor e desnutrição de uma população de risco de um bairro de Porto Alegre
}

\author{
Motor development and malnutrition in a risk population of a neighborhood in \\ Porto Alegre
}

Sabrina Scheid Mezzari ${ }^{1}$, Márcio Vinicius Fagundes Donadio², Laís Rodrigues Gerzson³, Carla Skilhan de Almeida ${ }^{4}$

\begin{abstract}
RESUMO
Objetivos: Verificar o perfil sociodemográfico de crianças moradoras de um bairro de periferia de Porto Alegre/RS - Brasil, com desnutrição ou com suspeita de desnutrição e se há uma associação do desenvolvimento neuropsicomotor com essas variáveis sociodemográficas. Métodos: Pesquisa retrospectiva, baseada em um estudo prévio na Unidade Básica de Saúde do Morro da Cruz na cidade de Porto Alegre/RS, com 169 crianças. Realizou-se uma avaliação antropométrica do peso/idade, estatura/idade e peso/estatura para o perfil nutricional; as variáveis das crianças no que se refere a sexo, idade, desenvolvimento neuropsicomotor pelo teste de triagem do Desenvolvimento de Denver II (TTDD-II) e, para as variáveis sociodemográficas, o número de moradores na casa, idade e escolaridade do cuidador, se a criança morava com a mãe ou com o pai, número de pessoas na casa que viviam com a criança, idade gestacional, prematuridade, peso ao nascimento, amamentação e o peso atual. Resultados: 45 apresentaram risco ou alto risco de desnutrição. As características demográficas e antropométricas, segundo sexo não apresentaram diferenças significativas quanto à idade, peso ao nascer e peso atual. Não houve associação significativa entre sexo e o desenvolvimento motor e linguagem. A escolaridade do cuidador e prematuridade não teve significância com o desenvolvimento neuropsicomotor. O desenvolvimento social apresentou associação significativa com o sexo masculino. Conclusões: o desenvolvimento social parece ser o mais acometido nas crianças com desnutrição ou alto risco de desnutrição e os meninos apresentam maior risco. Para esta amostra, parece não haver associação como prematuridade, peso ao nascer, idade gestacional, número de pessoas na casa, idade e escolaridade do cuidador.
\end{abstract}

Palavras-chave: Desenvolvimento Infantil. Criança. Desnutrição.

\begin{abstract}
Objectives: Verifying the sociodemographic profile of children living in a suburban neighborhood of Porto Alegre with malnutrition or suspected malnutrition and whether there was an association
\end{abstract}

1. Fisioterapeuta, Pontifícia Universidade Católica do Rio Grande do Sul (PUC-RS), Porto Alegre, RS, Brasil.

2. Pós-doutor em Fisiologia na Universidade de Edimburgo, Escócia. Professor do Curso de Fisioterapia da PUC-RS, Porto Alegre, RS, Brasil.

3. Programa de Pós-Graduação Saúde da Criança e do Adolescente, Universidade Federal do Rio Grande do Sul, Porto Alegre, RS, Brasil.

4. Departamento de Fisioterapia, Educação Física e Dança, Universidade Federal do Rio Grande do Sul, Porto Alegre, RS, Brasil.
CORRESPONDÊNCIA: Carla Skilhan de Almeida Universidade Federal do Rio Grande do Sul Rua Felizardo, 750, Jardim Botânico 90690-200, Porto Alegre, RS carlaskilhan@gmail.com

Recebido em: 20/03/2018 Aprovado em: 03/04/2019 
of neuropsychomotor development with these sociodemographic variables. Methods: Retrospective study, based on a previous study in the Basic Health Unit of Morro da Cruz - Porto Alegre/RS - Brazil, with 169 children. An anthropometric evaluation of weight/age, height /age and weight/height for the nutritional profile was carried out; age, neuropsychomotor development by the Denver Developmental Screening Test II (DDST-II) and, for sociodemographic variables, the number of residents in the house, age and schooling age and schooling of the legal guardian of the child, whether the child lived with the mother or father, number of people in the house who lived with the child, gestational age, prematurity, birth weight, breastfeeding, and current weight. Results: 45 children presented a risk/ high or risk of malnutrition. The demographic and anthropometric characteristics, according to gender, did not present significant differences regarding age, birth weight, and current weight. There was no significant association between sex and motor development and language. Caregiver education and prematurity were not significant with neuropsychomotor development. Social development was significantly associated with males. Conclusions: social development seems to be the most affected in children with malnutrition or high risk of malnutrition, and boys are at greater risk. For this sample, there seems to be no association such as prematurity, birth weight, gestational age, number of people in the house, age, and schooling of the caregiver.

Keywords: Child Development. Child. Malnutrition.

\section{INTRODUÇÃO}

Uma população é considerada de risco quando as famílias moram em regiões socialmente desfavorecidas.1,2 Estudos demonstram que, em populações de risco, a desnutrição está fortemente presente. ${ }^{3}$ A prevalência de desnutrição proteico-calórica, definida como "[...] deficiência energética e proteica com redução da massa muscular e adiposa $[\ldots]^{\prime \prime}$, vem reduzindo em crianças brasileiras menores de cinco anos. ${ }^{5} \mathrm{Na}$ Pesquisa Nacional de Demografia e Saúde da Criança e da Mulher (PNDS 2006), apenas 1,9\% das crianças nessa faixa etária apresentavam déficits de peso em relação à altura. ${ }^{5}$ Muitas vezes, existe a desnutrição por ingestão de alimentos pouco saudáveis. ${ }^{6}$

Entretanto, a desnutrição proteico-calórica ainda representa um problema de saúde públi$\mathrm{ca}^{7,8,9}$, posto que os danos sofridos no início da vida podem levar ao comprometimento permanente do indivíduo, inclusive afetando futuras gerações. ${ }^{7}$ A desnutrição ainda é considerada um dos fatores de maior risco de óbito infantil ${ }^{10,11}$, podendo afetar o crescimento físico e o desenvolvimento intelectual, pela interferência na saúde e nos níveis de energia necessários para o aprendizado. ${ }^{12}$

Associado à desnutrição, outros fatores podem alterar o desenvolvimento das crianças. Foi encontrado em alguns estudos ${ }^{13-17}$ que a escolaridade dos pais (possuem ensino médio incompleto ou menos), a idade do chefe de família (quando muito jovem), a situação de deficiência alimentar (pouca oferta de variedade alimentar), assistência à saúde deficitária, condições de moradia precária, um número maior de filhos, pouco estímulo ambiental, menor disponibilidade familiar para oferecer atenção à criança e renda familiar influenciam diretamente no desenvolvimento das crianças. ${ }^{18}$

Mas, entre os diferentes fatores sociodemográficos, a baixa renda familiar e a pobreza têm sido identificadas como os seus principais determinantes. De acordo com o Relatório do Programa das Nações Unidas ${ }^{19}$ para o desenvolvimento, cerca de 2,2 milhões de pessoas no mundo estão em situação de pobreza e mais de um terço da população se encontra a ponto de estar. Em condições extremas, a pobreza pode levar a uma ingestão insuficiente de alimentos e nutrientes. Além disso, segundo este mesmo relatório, entre as pessoas afetadas pela pobreza, 842 milhões tem crise de fome crônica, representando $12 \%$ da população mundial.

As diferenças biológicas, relacionadas ao sexo da criança também são marcadores de risco nutricional, com os meninos apresentando riscos mais elevados de desnutrição. ${ }^{20} \mathrm{~A}$ maior vulnerabilidade do sexo masculino nos primeiros anos de vida pode ser verificada também para outros desfechos como a mortalidade infantil que é maior nesse grupo de crianças. ${ }^{21}$

A idade do cuidador também é importante para o desenvolvimento da criança. Estudo reali- 
zado com o propósito de identificar as associações e os principais preditores do desenvolvimento motor e cognitivo de bebês filhos de mães adolescentes e adultas, observou diferenças significativas entre grupos nas variáveis de renda familiar $(p=0,011)$, tempo de aleitamento materno exclusivo $(p=0,017)$, cuidador/mãe ter trabalho remunerado fora de casa $(p<0,001)$, escolaridade materna $(p=0,003)$, escolaridade paterna $(p=0,013)$ e tempo de frequência em creche $(p<0,001) .{ }^{22} \mathrm{As}$ pesquisas ressaltam que mães mais novas oferecem menos brinquedos aos seus filhos, muito provavelmente devido às mães apresentarem menor conhecimento sobre o desenvolvimento infantil. Estudos ainda associam que mães jovens apresentam menores condições de cuidados aos seus bebês e restrições nas oportunidades de exploração ofertadas ao filho. ${ }^{23} \mathrm{~A}$ prematuridade da criança pode igualmente prejudicar o seu desenvolvimento motor, porque quanto mais prematura for a criança, maior o risco de ela apresentar atrasos de desenvolvimento motor. ${ }^{24}$

A avaliação do desenvolvimento assume papel destacado para essas crianças, sendo o fisioterapeuta um dos profissionais de saúde com importante papel. Muitas vezes, com o uso de escalas validadas e sensíveis, fisioterapeutas identificam atraso no desenvolvimento motor. ${ }^{25} \mathrm{~A}$ intervenção fisioterapêutica precoce costuma apresentar bons resultados, embora muitos bebês acabam sendo encaminhados tardiamente para acompanhamento, geralmente já apresentando algum tipo de deficiência, restringindo a intervenção e inviabilizando o alcance do objetivo de prevenção das alterações patológicas no desenvolvimento. ${ }^{26}$

Assim, é importante que a avaliação das características de crianças em risco de desenvolvimento e a sua associação com fatores que podem estar influenciando sejam feitas o mais precoce possível, possibilitando a promoção da saúde e a prevenção de problemas relacionados ao atraso no desenvolvimento motor. Portanto, este estudo enfatizou dois objetivos: 1. Verificar o perfil sociodemográfico de crianças moradoras de um bairro de periferia de Porto Alegre com desnutrição ou com suspeita de desnutrição (desnutrição/ suspeita); 2. Verificar se existe uma associação do desenvolvimento neuropsicomotor com essas variáveis sociodemográficas.

\section{METODOLOGIA}

Este foi um estudo retrospectivo, realizado através da consulta em prontuários de controle, em que os dados iniciais foram armazenados através de uma pesquisa do tipo observacional, analítico, transversal, prospectivo e controlado. 0 estudo utilizou o banco de dados da pesquisa intitulada "Vigilância e Educação em Saúde de Crianças Desnutridas e Obesas da Área Adstrita a uma Unidade Básica de Saúde do Município de Porto Alegre".

$O$ estudo foi realizado em uma grande comunidade de Porto Alegre (RS), por meio da Unidade Básica de Saúde (UBS) e Estratégia de Saúde da Família (ESF), e aprovado previamente pelo Comitê de Ética em Pesquisa da PUCRS (07/03556). O Termo de Consentimento Livre e Esclarecido (TCLE) foi formulado tomando por base a Resolução 466/12 do Conselho Nacional de Saúde e os responsáveis pelas crianças assinaram o TCLE.

A população em questão está cadastrada na comunidade Morro da Cruz, de 3.800 pessoas, e a principal fonte de renda familiar vem do comércio e do artesanato. É conhecida por ser um dos lugares mais violentos da cidade, onde o tráfico de drogas está presente em seu cotidiano. Apresenta condições sociais adversas como a falta de saneamento básico, precárias condições de moradia, dificuldade de acesso à educação e baixa escolaridade das famílias.

Previamente a este estudo, através da realização de um censo interdisciplinar, onde participaram estudantes e professores dos cursos de Enfermagem, Nutrição e Fisioterapia da Pontifícia Universidade Católica do Rio Grande do Sul, foram cadastradas na UBS todas as crianças com idade inferior a seis anos. Foi realizada uma avaliação antropométrica e calculados o escore $z$ dos índices peso/idade, estatura/idade e peso/estatura, tendo como padrão de referência as curvas de percentis do National Center for Health Statistics. ${ }^{27}$ A classificação do estado nutricional considerou os critérios da Organização Mundial da Saúde e Ministério da Saúde ${ }^{28}$, pelos quais é considerado desnutrição grave um escore $\mathrm{z}$ menor que - 3 ; moderada entre -2 e -3 e alto risco nutricional entre -1 e -2 desvios padrões da mediana. Crianças nascidas com idade gestacional abaixo de 37 semanas foram consideradas prematuras. ${ }^{29}$ 
A partir disso, 169 crianças foram recrutadas na comunidade por meio das agentes de saúde e consultas, todas as crianças foram avaliadas. Não houve recusa dos familiares em participar da pesquisa. As avaliações eram feitas ou no centro de saúde ou nas próprias casas das crianças, o que facilitou a adesão ao estudo. Destes, 45 (26,6\%) apresentaram desnutrição/suspeita e foram incluídas no estudo. Foram avaliadas as seguintes variáveis: sexo da criança; idade no momento da avaliação interdisciplinar; idade do cuidador (variável contínua); quem era o cuidador; a escolaridade do mesmo ( $\leq 8$ anos de estudo; $\geq 9$ anos de estudo); se a criança morava com a mãe ( $\operatorname{sim} x$ não); se a criança morava com o pai; o número de irmãos que viviam com a criança; a idade gestacional da criança ao nascimento (a termo e prematura < 37 IG); seu peso ao nascimento; se foi amamentado (sim ou não); seu peso atual no momento da coleta e o desenvolvimento motor, social e de linguagem.

Odesenvolvimento neuropsicomotor (DNPM) foi avaliado através do Teste de Triagem de Denver II adaptado para o português. ${ }^{30,31} \mathrm{O}$ teste é composto de 125 itens divididos em quatro domínios: 1. Pessoal-Social, avalia os aspectos da socialização da criança dentro e fora do ambiente familiar; 2. Linguagem, avalia a produção de som, capacidade de reconhecer, entender e usar a linguagem; 3. Motricidade Fina Adaptativa, avalia a coordenação do olho, da mão e manipulação de pequenos objetos e 4. Motricidade Ampla, avalia o controle motor corporal, sentar, caminhar, pular e todos os demais movimentos realizados pela musculatura ampla. Todos os itens são diretamente aplicados à criança e, se necessário, o responsável e/ou cuidador poderá informar se a criança realiza ou não determinada tarefa. Apenas o item de Motricidade Fina não foi avaliado.

Os casos indicativos de suspeita de atraso são aqueles em que a criança apresentava dois ou mais itens de atenção (a não realização da tarefa especificada quando 75 a $90 \%$ das crianças da faixa etária realizam) e/ou item de atenção somado a um item de falha (não realização do item quando 90 a $100 \%$ das crianças da faixa etária realizam), independentemente da área em que a falha ocorreu. Foram considerados casos indicativos de indícios de anormalidade aqueles em que a criança apresentou dois ou mais itens de falha.
A análise dos dados foi realizada no software Statistical Package for the Social Sciences (SPSS) versão 21.0. A análise descritiva univariada foi realizada por médias, desvios-padrão (para idade), frequências e proporções. A fim de verificar pressupostos da normalidade dos dados e a homogeneidade das variâncias entre os grupos, utilizou-se as provas de Shapiro-Wilk (não estavam normalmente distribuídos, $p=0,04$ ) e de Levene $(p>0,05)$. A análise das variáveis categóricas foi realizada através do Teste do Chi-quadrado de $\mathrm{Pe}$ arson e do Teste Exato de Fisher. Calculou-se o risco relativo para algumas das variáveis (RR). As variáveis numéricas distribuídas simetricamente foram comparadas pelo Teste não-paramétrico de Mann-Whitney, pois não havia uma apresentação normal da amostra. Foi adotado como significância estatística $p \leq 0,05$.

\section{RESULTADOS}

Participaram deste estudo 169 crianças, incluídas apenas as crianças que apresentaram desnutrição ou alto risco de desnutrição $(n=45)$. A média de idade da amostra foi de $3,2 \pm 1,7$ anos. Peso ao nascer foi $3,1 \pm 0,5 \mathrm{~kg}$ e peso atual $14,1 \pm 4,4 \mathrm{~kg}$. O cuidador apresentou uma média de idade de 29,1 $\pm 9,6$ anos. A média do número de moradores na mesma residência do participante foi de $4,8 \pm 1,5$ pessoas. $O$ número médio de irmãos foi de $2,1 \pm 1,6$. As demais variáveis da caracterização da amostra são mostradas na Tabela 1.

\section{Tabela 1}

Caracterização geral da amostra $(n=45)$

\begin{tabular}{lcc}
\hline & \multicolumn{2}{c}{ Amostra } \\
\hline Variável & $N=45$ & $(\%)$ \\
\hline $\begin{array}{l}\text { Idade Gestacional } \\
\text { (>37 semanas) }\end{array}$ & 38 & 84,4 \\
$\begin{array}{l}\text { Crianças amamentadas (no peito) } \\
\text { Grau de parentesco do cuidador }\end{array}$ & 40 & 88,9 \\
$\begin{array}{l}\text { (próprias mães) } \\
\text { Escolaridade do cuidador } \leq 8\end{array}$ & 36 & 80 \\
$\begin{array}{l}\text { de estudo (anos) } \\
\text { Moravam com suas mães }\end{array}$ & 35 & 77,8 \\
\hline
\end{tabular}

*Análise descritiva univariada com frequência e proporções Fonte: Produção dos autores. 
As características demográficas e antropométricas da amostra, segundo gênero não apresentaram diferenças significativas quanto à idade $(p=0,160)$, peso ao nascer $(p=0,869)$ e peso atual $(p=0,945)$. Além disso, foi analisado o desenvolvimento motor, social e linguagem. A associação entre o sexo das crianças e o desenvolvimento motor, social e de linguagem encontra-se na Tabela 2.

Tabela 2

Associação entre sexo e os desenvolvimentos motor, social e de linguagem na amostra $(n=45)$.

\begin{tabular}{|c|c|c|c|c|}
\hline & Masculino & Feminino & Total & $\mathrm{p}$ \\
\hline Variável & $n(\%)$ & $n(\%)$ & $n(\%)$ & \\
\hline \multicolumn{5}{|c|}{ Desenvolvimento motor } \\
\hline Normal & $20(100)$ & $22(88)$ & $42(93,3)$ & $0,242 *$ \\
\hline Suspeita/atraso & $0(0)$ & $3(12)$ & $3(6,7)$ & \\
\hline Total & $20(100)$ & $25(100)$ & $45(100)$ & \\
\hline \multicolumn{5}{|c|}{ Desenvolvimento social } \\
\hline Normal & $10(50)$ & $20(80)$ & $30(66,7)$ & $0,034 * *$ \\
\hline Suspeita/atraso & $10(50)$ & $5(20)$ & $15(33,3)$ & \\
\hline Total & $20(100)$ & $25(100)$ & $45(100)$ & \\
\hline \multicolumn{5}{|c|}{ Desenvolvimento linguagem } \\
\hline Normal & $16(80)$ & $23(92)$ & $39(86,7)$ & $0,383 *$ \\
\hline Suspeita/atraso & $4(20)$ & $2(8)$ & $6(13,3)$ & \\
\hline Total & $20(100)$ & $25(100)$ & $45(100)$ & \\
\hline
\end{tabular}

*Teste Exato de Fisher

**Teste do Chi-quadrado

$\mathrm{p}<0,05$

Fonte: Produção dos autores.

Não houve associação significativa entre sexo e o desenvolvimento motor, bem como entre o sexo e o desenvolvimento da linguagem. Contudo, o desenvolvimento social apresentou associação significativa com o sexo, onde a proporção de sujeitos do sexo masculino com suspeita e/ou atraso no desenvolvimento social foi maior, em comparação com o sexo feminino. A partir disso, calculou-se o risco relativo para o desenvolvimento social (igual a 4), ou seja, os indivíduos do sexo masculino tiveram quatro vezes mais chance de apresentarem alteração no desenvolvimento social (suspeita/atraso).

Analisando-se os dados relativos à associação entre escolaridade estratificada do cuidador e os desenvolvimentos motor, social e de linguagem na amostra investigada, apresentados na Tabela 3, verificou-se que não houve associação significativa entre escolaridade do cuidador e os desenvolvimentos motor, social e de linguagem. Da mesma forma, também não houve associação significativa entre prematuridade e o desenvolvimento motor, social e de linguagem, conforme apresentado na Tabela 4.

A comparação dos valores médios das características dos indivíduos da amostra e de seus cuidadores entre os diferentes desenvolvimentos é apresentada na Tabela 5. Não foram encontradas diferenças significativas entre nenhuma das variáveis investigadas e os diferentes desenvolvimentos (motor, social e linguagem). 
Tabela 3

Associação entre escolaridade estratificada do cuidador e os desenvolvimentos motor, social e de linguagem na amostra $(n=45)$.

\begin{tabular}{|c|c|c|c|c|c|c|c|}
\hline \multirow[b]{2}{*}{ Variável } & \multicolumn{2}{|c|}{$\leq 8$ anos } & \multicolumn{2}{|c|}{$\geq 9$ anos } & \multicolumn{2}{|c|}{ Total } & \multirow[t]{2}{*}{$p$} \\
\hline & $n$ & $(\%)$ & $n$ & $(\%)$ & $n$ & $(\%)$ & \\
\hline \multicolumn{8}{|c|}{ Desenvolvimento motor } \\
\hline Normal & 34 & $(97,1)$ & 8 & $(80)$ & 42 & $(93,3)$ & $0,119 *$ \\
\hline Suspeita/atraso & 1 & $(2,9)$ & 2 & $(20)$ & 3 & $(6,7)$ & \\
\hline Total & 35 & $(100)$ & 10 & $(100)$ & 45 & $(100)$ & \\
\hline \multicolumn{8}{|c|}{ Desenvolvimento social } \\
\hline Normal & 24 & $(68,6)$ & 6 & $(60)$ & 30 & $(66,7)$ & $0,710^{*}$ \\
\hline Suspeita/atraso & 11 & $(31,4)$ & 4 & $(40)$ & 15 & $(33,3)$ & \\
\hline Total & 35 & $(100)$ & 10 & $(100)$ & 45 & $(100)$ & \\
\hline \multicolumn{8}{|c|}{ Desenvolvimento linguagem } \\
\hline Normal & 32 & $(91,4)$ & 7 & $(70)$ & 39 & $(86,7)$ & $0,113^{*}$ \\
\hline Suspeita/atraso & 3 & $(8,6)$ & 3 & (30) & 6 & $(13,3)$ & \\
\hline Total & 35 & $(100)$ & 10 & $(100)$ & 45 & $(100)$ & \\
\hline
\end{tabular}

${ }^{*}$ Teste Exato de Fisher

$p<0,05$

Fonte: Produção dos autores.

Tabela 4

Associação entre a prematuridade e os desenvolvimentos motor, social e de linguagem na amostra $(n=45)$.

\begin{tabular}{|c|c|c|c|c|c|c|c|}
\hline \multicolumn{8}{|c|}{ Prematuro } \\
\hline \multirow[t]{2}{*}{ VARIÁVEL } & \multicolumn{2}{|c|}{ Sim } & \multicolumn{2}{|c|}{ Não } & \multicolumn{2}{|c|}{ Total } & \multirow[t]{2}{*}{$p$} \\
\hline & $n$ & $(\%)$ & $n$ & $(\%)$ & $n$ & $(\%)$ & \\
\hline \multicolumn{8}{|c|}{ Desenvolvimento motor } \\
\hline Normal & 6 & 85,7 & 36 & 94,7 & 42 & 93,3 & $0,405^{*}$ \\
\hline Suspeita/atraso & 1 & 14,3 & 2 & 5,3 & 3 & 6,7 & \\
\hline Total & 7 & 100 & 38 & 100 & 45 & 100 & \\
\hline \multicolumn{8}{|c|}{ Desenvolvimento social } \\
\hline Normal & 5 & 71,4 & 25 & 65,8 & 30 & 66,7 & $0,771^{*}$ \\
\hline Suspeita/atraso & 2 & 28,6 & 13 & 34,2 & 15 & 33,3 & \\
\hline Total & 7 & 100 & 38 & 100 & & & \\
\hline \multicolumn{8}{|c|}{ Desenvolvimento linguagem } \\
\hline Normal & 7 & 100 & 32 & 84,2 & 39 & 86,7 & $0,569 *$ \\
\hline Suspeita/atraso & 0 & 0 & 6 & 15,8 & 6 & 13,3 & \\
\hline Total & 7 & 100 & 38 & 100 & 45 & 100 & \\
\hline
\end{tabular}

*Teste Exato de Fisher

$p<0,05$

Fonte: Produção dos autores. 


\section{Tabela 5}

Comparação dos valores médios das características dos indivíduos da amostra e de seus cuidadores entre os diferentes desenvolvimentos $(n=45)$.

\begin{tabular}{|c|c|c|c|c|c|c|c|c|c|}
\hline \multirow[b]{2}{*}{ Variável } & \multicolumn{3}{|c|}{ Motor } & \multicolumn{3}{|c|}{ Social } & \multicolumn{3}{|c|}{ Linguagem } \\
\hline & Normal & $\begin{array}{l}\text { Suspeita } \\
\text { atraso }\end{array}$ & $p$ & Normal & $\begin{array}{l}\text { Suspeita } \\
\text { atraso }\end{array}$ & $p$ & Normal & $\begin{array}{l}\text { Suspeita } \\
\text { atraso }\end{array}$ & $p$ \\
\hline $\begin{array}{l}\text { Escolaridade } \\
\text { cuidador** }\end{array}$ & $6,4 \pm 2,6$ & $8,3 \pm 2,1$ & 0,212 & $6,4 \pm 2,8$ & $6,7 \pm 2,2$ & 0,664 & $6,3 \pm 2,5$ & $7,7 \pm 3,3$ & 0,242 \\
\hline Peso ao nascer** & $3,1 \pm 0,5$ & $3,1 \pm 0,4$ & 0,750 & $3,1 \pm 0,5$ & $3,1 \pm 0,6$ & 0,463 & $3,1 \pm 0,5$ & $3,3 \pm 0,5$ & 0,316 \\
\hline $\begin{array}{l}\text { N. }{ }^{\circ} \text { de pessoas } \\
\text { na casa** }\end{array}$ & $4,8 \pm 1,6$ & $5,3 \pm 0,6$ & 0,229 & $4,8 \pm 1,5$ & $4,9 \pm 1,6$ & 0,663 & $4,8 \pm 1,6$ & $5,2 \pm 1$ & 0,226 \\
\hline $\begin{array}{l}\text { Idade do } \\
\text { cuidador** }\end{array}$ & $29,4 \pm 29,9$ & $26,3 \pm 4,2$ & 0,766 & $30,7 \pm 10,1$ & $26,0 \pm 8$ & 0,212 & $29,5 \pm 9,3$ & $27 \pm 12,4$ & 0,545 \\
\hline $\begin{array}{l}\text { Idade } \\
\text { gestacional** }\end{array}$ & $40 \pm 2,5$ & $38 \pm 3,5$ & 0,228 & $39,7 \pm 2,6$ & $40,3 \pm 2,6$ & 0,374 & $39,6 \pm 2,7$ & $41,3 \pm 1,5$ & 0,123 \\
\hline \multicolumn{10}{|c|}{ Resultados expressos por médias \pm desvios-padrão } \\
\hline \multicolumn{10}{|c|}{ **Teste de Mann-Whitney } \\
\hline \multicolumn{10}{|c|}{$p<0,05$} \\
\hline Fonte: Produção dos & & & & & & & & & \\
\hline
\end{tabular}

\section{DISCUSSÃO}

Este estudo fez parte de um projeto maior, em que vários acadêmicos e profissionais da área da saúde estavam envolvidos, de forma interdisciplinar. Todas as medidas foram feitas no centro de saúde ou nas próprias casas que, sem dúvida, tinham um cenário de problemas visíveis de vulnerabilidade social. As crianças desnutridas/suspeita desse estudo alimentavam-se de forma inadequada, ingerindo salgadinhos e refrigerantes, por serem mais baratos, mais prático e de fácil acesso. A fisioterapia, para este estudo, verificou apenas os dados das crianças desnutridas e/ou com risco de desnutrição, comparando com o seu desenvolvimento. No entanto, foi uma amostra reduzida, ou seja, não comparamos as crianças sem risco de desnutrição e/ou desnutridas com o seu desenvolvimento, o que limitou especificamente este estudo nos seus resultados.

Sabe-se que o desenvolvimento da criança é de extrema importância, pois a prepara para seu futuro na sociedade. Assim, observa-se que o desenvolvimento acontece por ações integradas do organismo, trazendo influências para o processo maturacional, para o processamento das informações, preparando a criança para agir e interagir, viabilizando oportunidades no desenvolvimento linguístico, social, cognitivo e motor. ${ }^{32}$
Sendo assim, a falta de alimentação adequada diminui a disponibilidade e prejudica $o$ engajamento do ser humano em suas atividades cotidianas e intelectuais. No caso da extrema pobreza, dificilmente há padrões de desempenho para garantir uma alimentação saudável. A presença do alimento e a assiduidade alimentar são irregulares, afetando o estabelecimento de hábitos e rotinas relativas à alimentação. Assim, compromete o desenvolvimento num contexto geral. Nesse sentido, compreende-se a relação entre o estado de desnutrição e a falta de êxito nas tarefas de autocuidado, função social e mobilidade em crianças com quadro de desnutrição. A desmotivação para explorar o ambiente soma-se ao pouco estímulo que o ambiente oferece, concorrendo para o mau desempenho das crianças. ${ }^{33}$

Uma das nossas expectativas foi que poderia existir diferença de desenvolvimento entre os gêneros ${ }^{34}$ nas crianças desnutridas/suspeita, como existe em estudo prévio. Em nossos achados com crianças desnutridas/suspeita, os meninos não só apresentam mais suspeita/atraso no desenvolvimento social do que as meninas, como tem chance de ser quatro vezes maior para o atraso social. Isto pode ser explicado porque os meninos têm biologicamente maior risco de transtornos do desenvolvimento no útero que as meni- 
nas, em função das cargas hormonais intra-útero. Cientistas britânicos mediram o hormônio sexual masculino (testosterona) no líquido amniótico e descobriram que os níveis estavam relacionados tanto com o desenvolvimento de autismo como o de transtornos de linguagem. $O$ estudo aponta que meninos têm geralmente o desenvolvimento da linguagem um pouco mais tardio que as meninas, podendo estar relacionados com seu desenvolvimento social. No estudo, existe uma porcentagem de meninos com suspeita de atraso de linguagem maior do que meninas, o que pode relacionar-se com esse aspecto social. Mais ainda, a desnutrição pode acentuar estas diferenças entre os gêneros no que se refere à linguagem e aos aspectos sociais. ${ }^{35}$

Pensando sobre o desenvolvimento infantil como um sistema aberto, dinâmico e complexo, este resultado pode estar relacionado com o contexto de interesse por parte dos meninos e meninas, isto é, meninos se interessam por atividades mais egocêntricas, agindo de maneira competitiva e individualizada, inclusive em suas próprias relações de aprendizagem, enquanto as meninas são mais sociáveis, cooperativas e frequentemente mais estimuladas a participarem de atividades mais calmas. ${ }^{36}$

Neste estudo observou-se que não houve associação entre a escolaridade do cuidador e o desenvolvimento motor, social e de linguagem das crianças desnutridas/suspeita, contrariando o que é relatado na literatura, talvez devido a amostra reduzida. Atrasos no desenvolvimento infantil têm sido associados a fatores como as características ambientais, individuais e demográficas das famílias. Apesar disso, o baixo grau de escolaridade materna se destaca nas pesquisas como importante fator de risco para o atraso no desenvolvimento infantil. A chance de uma mãe, com tempo de escolaridade menor que quatro anos, ter um filho com desnutrição é três vezes maior do que para mães com escolaridade superior. Deste modo, condições sociais, educacionais e econômicas adversas da família são fatores que podem contribuir para o inadequado crescimento infantil, refletindo-se em déficit nutricional. $37,38,39,40$

A figura feminina foi predominante entre os cuidadores avaliados nesta pesquisa, concordante com a literatura, enfatizando a tradição histórica e cultural da mulher em assumir o principal papel do ato de cuidar. Essa divisão sexual entre os cuidadores parece amparada na vivência da maternidade, determinando assim que as mulheres estariam mais direcionadas a atividades de cuidado, ensinada às mulheres dentro da própria família, através das gerações. Sendo assim, o papel da mãe no cenário familiar é o de detentora do cuidado integral que não poupa esforços para garantir o melhor aos filhos. Atualmente, embora tenham ocorrido mudanças nas famílias, dificilmente o homem se responsabiliza pelos cuidados diretos e geralmente os mesmos participam do cuidado por meio do sustento da família. ${ }^{41}$

Um estudo realizado por Saldan et al. ${ }^{42}$ pode verificar que as crianças desnutridas estavam expostas aos fatores de risco como o fato de serem filhos de mães adolescentes, baixa escolaridade materna, número de filhos, ausência do pai no domicílio e renda insuficiente. O mesmo relata que as pessoas envolvidas nos momentos da alimentação e cuidados da criança foram as mães e as avós. O preparo das refeições e o cuidado dos filhos ainda estão diretamente ligados ao universo feminino nas camadas populares e é um reflexo da divisão sexual do trabalho. Neste estudo as mães não trabalhavam fora do lar, algumas residiam na casa dos seus filhos, outras coabitavam com o esposo que trabalhava fora e era o provedor, e outras moravam apenas com o filho. Além disso, não foi possível visualizar a participação dos pais e avôs no cuidado das crianças. A adesão dos homens à arte de cozinhar se restringe a ocasiões especiais e o preparo das refeições continua como tarefa feminina. Mesmo sendo a mãe a principal cuidadora, observamos que as crianças desnutridas/suspeita se alimentavam de forma inadequada (ingerindo salgadinhos e refrigerantes), sem horários definidos e sem a alimentação saudável.

Rodrigues et al. ${ }^{17}$ viram em seu estudo que houve associação entre a suspeita de atraso no desenvolvimento com o número de pessoas na casa, confirmando que nas famílias com maior número, em geral, há menos estímulos para que as crianças possam explorar seu potencial de desenvolvimento. Embora não ocorreu resultado significativo neste item da Tabela 5, a média do número de pessoas é maior nos casos de atraso/suspeita. 
Esse menor estímulo provavelmente está associado à menor disponibilidade materna para oferecer atenção à criança. Ainda, Eickmann et al. ${ }^{43}$ citam em seu estudo a importância do vínculo entre mãe e criança ${ }^{44}$, ressaltando que, no domicílio, a proporção adulto/criança é, em geral, menor do que na creche, propiciando contato mais próximo da criança com uma pessoa de referência, o que é importante para o desenvolvimento infantil. ${ }^{45}$

As limitações do estudo incluem tamanho da amostra, ausência do grupo controle para que assim pudesse realizar as comparações necessárias e a continuidade do estudo para contribuir no esclarecimento dos aspectos importantes. Outra limitação foi de que não foram utilizados testes de tamanho do efeito nas comparações que complementaria o entendimento dos resultados.

\section{CONCLUSÃO}

O estudo indica que o desenvolvimento social parece ser o mais acometido nas crianças com desnutrição ou alto risco de desnutrição e que os meninos apresentam maior risco em comparação com as meninas. No entanto, para esta amostra, parece não haver associação com fatores importantes como prematuridade, peso ao nascer, idade gestacional, número de pessoas na casa, idade e escolaridade do cuidador. Isto aponta para uma maior relevância dos aspectos sociais nessas crianças, podendo servir como base para intervenções de saúde pública que beneficiem as famílias e a sociedade como um todo. Portanto, fica um convite aos fisioterapeutas para que pensem em estratégias avaliativas e interventivas para as crianças com desnutrição ou com suspeita de nutrição já que elas estão em pleno ápice do seu desenvolvimento neuropsicomotor.

\section{REFERÊNCIAS}

1. Jesus GM de, Castelão ES, Vieira T de O, Gomes DR, Vieira GO. Déficit nutricional em crianças de uma cidade de grande porte do interior da Bahia, Brasil. Cien Saude Colet. 2014;19(5):1581-8. Available at: http://www.scielo.br/scielo.php?script=sci_arttext\&pi$\mathrm{d}=\mathrm{S} 1413-81232014000501581 \& \mathrm{Ing}=\mathrm{pt \&}$ tIng $=\mathrm{pt}$

2. Almeida P, Saab É, Santos DN, Santos LM dos, Santos CADST AA. Desenvolvimento de lactentes em uma cidade do Interior da Bahia: aspectos nutricionais e psicossociais. Pediatr Mod. 2012;48(3).

3. Marramarco CA, Krebs RJ, Valentini NC, Ramalho MH da S, Santos JOL dos, Nobre GC. Crianças desnutridas pregressas, com sobrepeso e obesas apresentam desempenho motor pobre. Rev da Educ Física/UEM. 2012;23(2):17582. Available at: http://periodicos.uem.br/ojs/index. php/RevEducFis/article/view/13002

4. Ribeiro CCC, Silva MCB da, Machado CMP, Ribeiro MRC, Thomaz ÉBAF. A gravidade da cárie está associada à desnutrição proteico-calórica em pré-escolares? Cien Saude Colet. 2014;19(3):957-65. Available at: http://www.scielo.br/scielo.php?script=sci_arttext\&pi$d=S 1413-81232014000300957 \&$ Ing $=p t \& t \mid n g=p t$

5. Ministério da Saúde. Pesquisa Nacional de Demografia e Saúde da Criança e da Mulher - PNDS 2006: Dimensões do processo reprodutivo e da saúde da criança. 2009. Available at: http://bvsms.saude.gov.br/bvs/publicacoes/pnds_crianca_mulher.pdf

6. Claro RM, Santos MAS, Oliveira TP, Pereira CA, Szwarcwald CL, Malta DC. Consumo de alimentos não saudáveis relacionados a doenças crônicas não transmissíveis no Brasil: Pesquisa Nacional de Saúde, 2013. Epidemiol e Serviços Saúde. 2015;24(2):257-65. Available at: http://www.scielo. $\mathrm{br} /$ scielo.php?pid=S2237-96222015000200257\&script=sci_abstract\&tlng $=$ pt

7. Victora CG, Adair L, Fall C, Hallal PC, Martorell R, Richter $\mathrm{L}$, et al. Maternal and child undernutrition: consequences for adult health and human capital. Lancet. 2008;371(9609):340-57. Available at: https://www.thelancet.com/action/showPdf?pii=S0140-6736\%2807\%2961692-4

8. Onis M de, Blössner M, Borghi E. Prevalence and trends of stunting among pre-school children, 1990-2020. Public Health Nutr. 2012;15(1):142-8. Available at: https:// www.cambridge.org/core/services/aop-cambridge-core/ content/view/6FDF3AC29E66FD148917CE2B26B84B2D/ S1368980011001315a.pdf/prevalence_and_trends_of_ stunting_among_preschool_children_19902020.pdf

9. Gondim SSR, Diniz A da S, Cagliari MPP, Araújo E de S, Queiroz D de, Paiva A de A. Relação entre níveis de hemoglobina, concentração de retinol sérico e estado nutricional em crianças de 6 a 59 meses do Estado da Paraíba. Rev Nutr. 2012;25(4):441-9. Available at: http://www.scielo.br/scielo.php?script=sci_arttext\&pi$\mathrm{d}=\mathrm{S} 1415-52732012000400002 \& \mathrm{lng}=\mathrm{pt} \& \mathrm{t} \mid \mathrm{ng}=\mathrm{pt}$

10. Black RE, Allen LH, Bhutta ZA, Caulfield LE, de Onis $M$, Ezzati $M$, et al. Maternal and child undernutrition: global and regional exposures and health consequences. Lancet. 2008;371(9608):243-60. Available at: https://linkinghub.elsevier.com/retrieve/pii/S0140673607616900

11. Cruz MCC da, Leite I da C. Fatores de risco para déficits estaturais no segundo ano de vida : Brasil , PNDS , 1996. Rev Bras Estud Popul. 2002;19(1):131-40. Available at: https://www.rebep.org.br/revista/article/view/334/pdf_314

12. Leal VS, Lira PIC de, Menezes RCE de, Oliveira JS, Costa EC, Andrade SLLS de. Desnutrição e excesso de 
peso em crianças e adolescentes: uma revisão de estudos brasileiros. Rev Paul Pediatr. 2012;30(3):415-22. Available at: http://www.scielo.br/scielo.php?script=sci_arttext\&pid=S0103-05822012000300017\&lng=pt\&tlng=pt

13. Barros FC, Victora CG, Scherpbier R, Gwatkin D. Socioeconomic inequities in the health and nutrition of children in low/middle income countries. Rev Saude Publica. 2010;44(1):1-16. Available at: http:// www.scielo.br/scielo.php?script $=$ sci_arttext\&pi$\mathrm{d}=$ S0034-89102010000100001\&lng=en\&tIng =em

14. Lima ALL de, Silva ACF da, Konno SC, Conde $W L$, Benicio MHDA, Monteiro CA. Causas do declínio acelerado da desnutrição infantil no Nordeste do Brasil (19861996-2006). Rev Saude Publica. 2010;44(1):17-27. Available at: http://www.scielo.br/pdf/rsp/v44n1/02.pdf

15. Onis M, Blössner M, Borghi E, Frongillo EA, Morris R. Estimates of Global Prevalence of Childhood Underweight in 1990 and 2015. JAMA. 2004;291(21):2600-2606.

16. Chagas DC das, Silva AAM da, Batista RFL, Simões VMF, Lamy ZC, Coimbra LC, et al. Prevalência e fatores associados à desnutrição e ao excesso de peso em menores de cinco anos nos seis maiores municípios do Maranhão. Rev Bras Epidemiol. 2013;16(1):146-56. Available at: http://www.scielo.br/scielo.php?script=sci_arttext\&pi$\mathrm{d}=$ S1415-790X2013000100146\&lng =pt\&tIng =pt

17. Rodrigues CO, Freitas AS, Freitas ALR, Júnior EPF, Miranda JA. Prevalência de obesidade, sobrepeso e nível sócio-econômico em escolares de 6 a 10 anos da cidade de Montes Claros - MG. Motricidade. 2012;8(2):462-469. Available at: http://www.redalyc. org/pdf/2730/273023568055.pdf

18. Mansur $S$, Neto F. Desenvolvimento neuropsicomotor de lactentes desnutridos. Rev Bras Fisioter. 2006;10(2):185-91. Available at: http:// www.scielo.br/scielo.php?script $=$ sci_arttext\&pi$\mathrm{d}=\mathrm{S} 1413-35552006000200008 \& \mathrm{lng}=\mathrm{pt} \& \mathrm{nrm}=\mathrm{iso} \& \mathrm{t} \operatorname{lng}=\mathrm{pt}$

19. Brasil. Atlas do Desenvolvimento Humano no Brasil. Brasília: PNUD; 2014.

20. Akombi BJ, Agho KE, Hall JJ, Merom D, Astell-Burt T, Renzaho AMN. Stunting and severe stunting among children under- 5 years in Nigeria: A multilevel analysis. BMC Pediatr. 2017;17(1):1-16. Available at: https:// bmcpediatr.biomedcentral.com/track/pdf/10.1186/ s12887-016-0770-z

21. Barros AJD, Victora CG. Measuring Coverage in $\mathrm{MNCH}$ : Determining and Interpreting Inequalities in Coverage of Maternal, Newborn, and Child Health Interventions. Madise N, organizador. PLoS Med. 2013;10(5). Available at: https://dx.plos.org/10.1371/journal.pmed.1001390

22. Borba LS de, Pereira KRG, Valentini NC. Motor and cognitive development predictors of infantis of adolescents and adults mothers. J Phys Educ. 2017;28(1):1-16. Available at: http://www.scielo.br/pdf/jpe/v28/24482455-jpe-28-e2811.pdf

23. Saccani $R$, Valentini $N C$, Pereira $K R$, Müller $A B$, Gabbard $C$. Associations of biological factors and affordances in the home with infant motor development. Pediatr Int. 2013;55(2):197-203.
24. Maia PC, Silva LP, Oliveira MMC, Cardoso MVLML. Desenvolvimento motor de crianças prematuras e a termo - Uso da Alberta Infant Motor Scale. ACTA Paul Enferm. 2011;24(5):670-5. Available at: http://www.scielo.br/pdf/ape/v24n5/12v24n5.pdf

25. Melo FR, Pereira A. Inclusão escolar do aluno com deficiência física: visão dos professores acerca da colaboração do fisioterapeuta. Rev Bras Educ Espec. 2013;19(1):93-106. Available at: http://www.scielo.br/pdf/rbee/v19n1/07.pdf

26. Formiga CKMR, Pedrazzani ES, Tudella E. Desenvolvimento motor de lactentes pré-termo participantes de um programa de intervenção fisioterapêutica precoce. Rev bras fisioter. 2004;8(3):239-245. Available at: file://C:/Users/Marcos/Downloads/Desenvolvimento_ motor_de_lactentes_pre-termo_parti.pdf

27. World Health Organization. Multicentre Growth Reference Study Group. WHO child growth standards: length/height-for-age, weight-for-age, weight-for-length, weight-for-height and body mass index-for-age: methods and development. Geneva: WHO; 2006. 321 p. Available at: https:// www.who.int/childgrowth/standards/Technical_report.pdf

28. Ministério da Saúde. Incorporação das curvas de crescimento da Organização Mundial da Saúde de 2006 e 2007 no SISVAN. Available at: http://www.sprs.com.br/ sprs2013/bancoimg/131209104419oms2006_2007.pdf

29. Lotto CR, Linhares MBM. Contato "pele a pele" na prevenção de dor em bebês prematuros: revisão sistemática da literatura. Temas em Psicol. 2018;26(4):1699-713. Available at: http://pepsic.bvsalud.org/pdf/tp/v26n4/v26n4a01.pdf

30. Pinto FC de $A$, Isotani SM, Sabates, AL, Perissinoto, J. Denver II: comportamentos propostos comparados aos de crianças paulistanas. Rev. CEFAC. 2015;17(4):1262-9. Available at: http://www.scielo.br/ scielo.php?pid =S1516-18462015000401262\&script $=$ sci_abstract\&tlng=pt

31. Sabatés AL, Lamônica DAC, Perissinoto J, Brêtas JS, Silva MGB, Rezende MA, et al. Teste de triagem do desenvolvimento Denver II: adaptação transcultural para a criança brasileira. Com autorização do autor Frankenburg WK. São Paulo; 2013.

32. Madaschi V, Mecca TP, Macedo EC, Paula CS. Bayley-III Scales of Infant and Toddler Development: Transcultural Adaptation and Psychometric Properties. Paid (Ribeirão Preto). 2016;26(64):189-97. Available at: http://www.scielo.br/scielo.php?script=sci_arttext\&pi$\mathrm{d}=$ S0103-863X2016000200189\&Ing=en\&tIng=em

33. Silva $M C$ da, Silva ÂCD da. Desempenho funcional de crianças com desnutrição crônica na faixa etária de um a três anos. Cad Ter Ocup da UFSCar. 2014;22(2):327-34. Available at: http://doi.editoracubo.com.br/10.4322/cto.2014.054

34. Pinto EB, Vilanova LCP, Vieira RM. O desenvolvimento do comportamento da criança no primeiro ano de vida: padronização de uma escala para avaliação e acompanhamento. São Paulo: FAPESP; 1997.

35. Norwegian Institute of Public Health. (2014, February 17). Gender, genes play important role in de- 
layed language development. ScienceDaily. Retrieved May 22, 2019 from https://www.sciencedaily.com/releases/2014/02/140217085246.htm

36. Oliveira D da $S$, Oliveira IS de, Cattuzzo MT. A influência do gênero e idade no desempenho das habilidades locomotoras de crianças de primeira infância. Rev Bras Educ Física e Esporte. 2013;27(4):647-55. Available at: http://www.scielo.br/scielo.php?script=sci_arttext\&pi$\mathrm{d}=$ S1807-55092013000400012\&lng=pt\&nrm=iso\&tIng=em

37. Breigeiron MK, Miranda MN de, Souza AOW de, Gerhardt LM, Valente MT, Witkowski MC. Associação entre estado nutricional, aleitamento materno exclusivo e tempo de internação hospitalar de crianças. Rev Gaúcha Enferm. 2015;36(spe):47-54. Available at: http://www.scielo.br/scielo.php?script=sci_arttext\&pi$\mathrm{d}=$ S1983-14472015000500047\&Ing=pt\&tIng=pt

38. Amaro LL de M, Pinto SA, Morais RL de $S$, Tolentino JA, Felício LR, Camargos ACR, et al. Desenvolvimento Infantil : Comparação entre crianças que frequentam ou não Creches Públicas. J Hum Growth Dev. 2015;25(2):170-6. Available at: http:// pepsic.bvsalud.org/scielo.php?script $=$ sci_arttext\&pi$\mathrm{d}=$ S0104-12822015000200006\&lng =pt\&nrm=iso\&tlng=pt

39. Defilipo ÉC, Frônio J da S, Teixeira MTB, Leite ICG, Bastos RR, Vieira M de $T$, et al. Oportunidades do ambiente domiciliar para o desenvolvimento motor. Rev Saude Publica. 2012;46(4):633-41. Available at: http://www.scielo.br/scielo.php?script=sci_arttext\&pi$d=$ S0034-89102012000400007

40. Ramos CV, Dumith SC, César JA. Prevalence and factors associated with stunting and excess weight in children aged 0-5 years from the Brazilian semi-arid region. J Pediatr (Rio J).2015;91(2):175-82. Available at: https://www.sciencedirect.com/science/article/pii/ S2255553615000166?via\%3Dihub

41. Barros ALO, Barros AO, Barros $G L$ de $M$, Santos MTBR. Sobrecarga dos cuidadores de crianças e adolescentes com Síndrome de Down. Cien Saude Colet. 2017;22(11):3625-34. Available at: http:// www.scielo.br/scielo.php?script $=$ sci_arttext\&pi$\mathrm{d}=\mathrm{S} 1413-81232017021103625 \& \mathrm{ng}=\mathrm{pt} \& \mathrm{t} \mid \mathrm{ng}=\mathrm{pt}$

42. Saldan PC, Demario RL, Brecailo MK, Ferriani M das GC, Mello DF de. Interaction during feeding times between mothers and malnourished children under two years of age. Cien Saude Colet. 2015;20(1):65-74. Available at: http://www.scielo.br/scielo.php?script=sci_arttext\&pi$\mathrm{d}=\mathrm{S} 1413-81232015000100065 \& \mathrm{lng}=$ en\&tIng $=e \mathrm{~m}$

43. Eickmann SH, Maciel AMS, Lira PIC, Lima M de C. Fatores associados ao desenvolvimento mental e motor de crianças de quatro creches públicas de Recife, Brasil. Rev Paul Pediatr. 2009;27(3):282-8. Available at: http://www.scielo.br/scielo.php?script=sci_arttext\&pi$\mathrm{d}=\mathrm{S} 0103-05822009000300008 \& \mathrm{lng}=\mathrm{pt} \& \mathrm{nrm}=\mathrm{iso} \& \mathrm{t}$ Ing $=\mathrm{pt}$

44. Nardi CG de A, Rodrigues OMPR, Melchiori LE, Salgado MH, Tavano LD. Bebês com Sequência de Pierre Robin: saúde mental materna e interação mãe-bebê. Estud Psicol. 2015;32(1):129-40. Available at: http://www.scielo.br/scielo.php?script=sci_arttext\&pi$\mathrm{d}=\mathrm{S} 0103-166 \times 2015000100129 \& \mathrm{Ing}=\mathrm{pt} \& \mathrm{t} \operatorname{lng}=\mathrm{pt}$

45. Dourado JS, Carvalho AS da S, Lemos SMA. Development of communication of children aged between one and three years old and their relationship with the family and school environments. Rev CEFAC. 2015;17(1):8899. Available at: http://www.scielo.br/pdf/rcefac/ v17n1/en_1982-0216-rcefac-17-01-00088.pdf 